\title{
Tuberculosis in maintenance haemodialysis patients. Study from an endemic area
}

\author{
K. K. Malhotra* \\ M.D., M.A.M.S. \\ U. N. BHUYAN $\dagger$ \\ M.D.
}

\author{
M. K. PARASHAR* \\ M.D.
}

S. C. DASH*
M.D.. D.M.
R. K. SHARMA* M.D.

R. KUMAR*

M.D., M.R.C.P.

\author{
D. S. RANA* \\ M.D. \\ Departments of *Medicine (Renal Division) and $\dagger$ Pathology, All India Institute of Medical Sciences, \\ New Delhi-110029, India
}

\begin{abstract}
Summary
One hundred and fifty patients requiring maintenance haemodialysis were investigated to determine the incidence and pattern of tuberculosis. Twenty patients were found to have tuberculosis, giving an incidence of 13.3 times that of the general population. The most frequent clinical presentations of tuberculosis in these patients were pyrexia, pleural effusion and lymphadenopathy. Diagnostic difficulties were encountered in 7 patients in whom therapeutic trial with anti-tubercular drugs had to be undertaken. Two patients died from tuberculosis. Five patients received renal transplants after initial treatment of tuberculosis.
\end{abstract}

\section{Introduction}

Host resistance to infection is primarily mediated by cellular immunity which is deficient in patients with chronic renal failure (Mackaness, 1971; Malaviya et al., 1973). The uraemic patients have impairment of delayed hypersensitivity skin reactions and prolonged skin homograft survival (Wilson, Kirkpatrick and Talmage, 1965; Boulton-Jones et al., 1973; Nakhla and Goggin, 1973; Dammin, Cough and Murray, 1957). The anergy of such patients has been attributed to either chronic renal insufficiency or to their overwhelming tubercular infection (Munt, 1971). Ogg, Toseland and Cameron (1968) were among the earlier workers to report the occurrence of tuberculosis in a patient on haemodialysis. Pradhan et al. (1974) reported increased frequency of tuberculosis in patients on maintenance haemodialysis (MHD) and this interested several dialysis centres to look into the problem. The clinical manifestations of tuberculosis in uraemia may be atypical and its diagnosis may presento challenge to the clinicians. Tuberculosis is endenapc in India and it was thought worthwhile to study its incidence and the pattern of the disease in patiens with chronic renal failure who developed tubercufosis during the course of MHD.

\section{Materials and methods}

During the period from January 1975 to June 1980 , 150 patients have been on MHD at the Dialysis Unt of the All India Institute of Medical Sciencess Hospital, New Delhi. The chronic dialysis programme at this Unit is predominantly transplant-orientated. Twenty out of these 150 patients were found to have developed tuberculosis during the course of MHB. There were 15 males and 5 females, their age ranged from 20 to 54 years. The basic renal disease in the patients was as follows: chronic glomerulonephritis (CGN), 15 cases; chronic pyelonephritis (CPN), one case; renal calculi with secondary CPN, 2 cases; gout with CPN, one case; diabetic glomerulo sclerosis, one case. The specific investigations included radiological studies; cultures of sputumi, urine, pleural fluid, ascitic fluid and cerebrospinal fluid for acid-fast bacillus (AFB); biopsies of lymph node, pleura, liver and bone. Specimens from abdominal laparotomy were available in one case and post-mortem specimens were available In another. Whenever the clinical picture was suggestize of tuberculosis but a definitive diagnosis could net be substantiated by investigation, a therapeutic trigl with antitubercular drugs was undertaken andoga presumptive diagnosis made in those patients sho ing a good therapeutic response. 


\section{Results}

The main clinical features and results of special investigations are given in Table 1. The duration of renal failure as judged from past medical records and development of uraemic symptoms varied from 2 to 30 months. It is likely that the renal failure was of longer duration in some of the cases. The duration of MHD before the recognition of tuberculosis varied from one week to 9 months in 18 patients. Tuberculosis was detected at the time of onset of haemodialysis in the remaining 2 patients. Two patients had a past history of tuberculosis, one (no. 9) had had pulmonary tuberculosis 15 years before and the other (no. 20) had had cervical lymphadenopathy and tuberculoma of the brain one year before the onset of chronic renal failure.

The clinical features of all cases are summarized in Table 2.

\section{Cultures}

Cultures for AFB were obtained from sputum, pleural fluid, ascitic fluid, urine and CSF whenever indicated. There were 4 cases with pulmonary involvement; sputum, however, was available only in 3. The sputum was positive for AFB in one patient (no. 15) and negative in the remaining 2 (nos 2 and 9). The pleural fluid was positive for AFB culture in only 2 of the 10 cases. Ascitic fluid cultures in the ascites cases were repeatedly negative in both. One of the cases with ascites (no. 6) showed tubercles on the peritoneum, liver and spleen. Cultures of urine, CSF, and bone abscess were done in one case each, and the first 2 were negative.

\section{Histology}

The peripheral lymph nodes were available for biopsy in 6 patients and showed evidence of tuberculosis in all cases. The pleural biopsy was compatible with tubercular granuloma in 2 of the 5 cases where it was done. Liver biopsy was done for evidence of tuberculosis in 2 cases with hepatomegaly and ascites; it was negative in both. Postmortem examination of case no. 1 , who had shown tubercular cervical lymphadenopathy in life, revealed histological evidence of tuberculosis of tracheobronchial and hilar lymph nodes.

\section{Diagnosis}

The diagnosis of tuberculosis was considered definite if there was positive culture for Mycobacterium tuberculosis or a classical histological picture of tubercular granuloma on histology specimens. Thirteen of 20 patients fulfilled such criteria for 'definite diagnosis'. In the remaining 7 cases the diagnosis of tuberculosis was considered 'probable'. There was unequivocal clinical improvement after a therapeutic trial with antituber- cular drugs despite the absence of bacteriological or histological proof in these 7 cases. Table 3 shows the diagnostic yield of tuberculosis by various methods. Five patients subsequently underwent renal transplantation after initial treatment with antitubercular drugs for periods varying from 3 to 5.5 months. One patient died one week after renal transplantation because of acute rejection, and tuberculosis of the tracheo-bronchial and hilar lymph nodes was found at post-mortem.

\section{Antitubercular therapy}

All patients received a combination of 2 antitubercular drugs, isoniazid $200 \mathrm{mg} /$ day and rifampicin $10 \mathrm{mg} / \mathrm{kg}$ body weight per day. Ethambutol was used in addition in some of the cases where tubercular disease was more widespread and more acute. Its dose had to be modified because of renal failure, the usual dose employed was $7.5 \mathrm{mg} / \mathrm{kg}$ body weight per day. The antitubercular drugs were given in a single dose in the evening. On days of dialysis, the drugs were given at the end of dialysis. The patients awaiting renal transplantation were given a minimum of 3 months of antitubercular treatment before surgery, some had to be treated for longer periods to achieve control of active disease. The antitubercular treatment was continued for a minimum period of 2 years.

\section{Mortality}

Twelve out of 20 patients died during the period of observation. Death was due to tuberculosis in only 2 cases. Six patients died from the consequences of renal failure or hypertension; in 4 of these, dialysis treatment was irregular and inadequate owing to financial limitations. One patient died from extensive gastrointestinal bleeding, one from fulminant chest infection and deaths of 2 transplant recipients were due to rejection. Another 3 transplant recipients in this series are doing well and follow-up after surgery in these 3 cases has been 27 months, 15 months and 3 months to date (March 1981). Five patients are doing well on MHD.

\section{Discussion}

Tuberculosis is a serious health problem in India. The incidence of tuberculosis in children and adults in India has been reported to be about $1 \%$ (National Tuberculosis Institute, 1974) and $1.31 \%$ in a recent survey in Delhi (Goyal, Mathur and Pamra, 1978). Tuberculosis was found in $13.3 \%$ of the present MHD patients. It is obvious that patients with chronic renal failure on MHD run a very high risk of developing tuberculosis with an incidence varying from 6 to 16 times that of the general population as has been reported from many countries (Papadimitriou, Memmos and Metaxas, 1979; Amedia 
K. K. Malhotra et al.

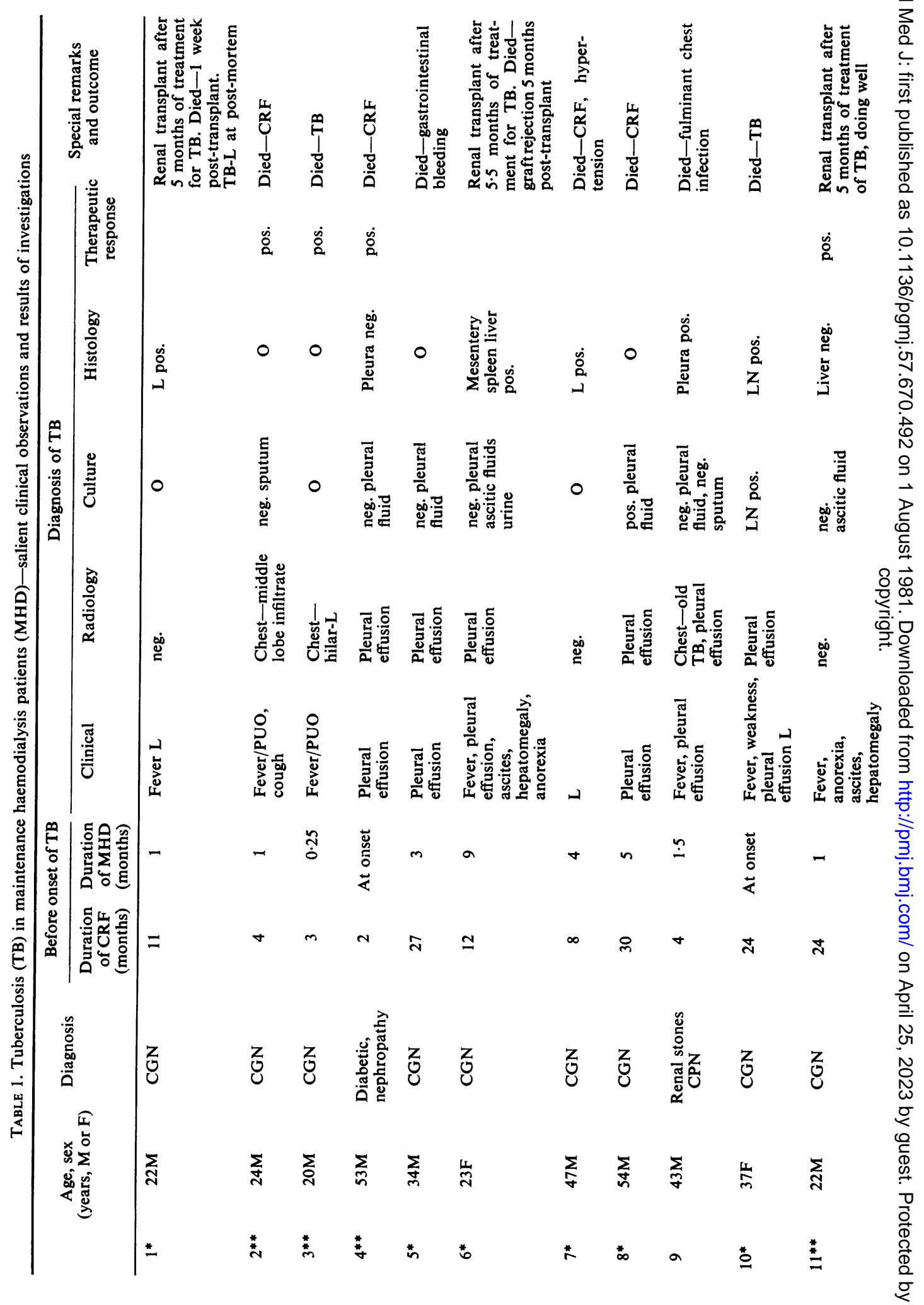




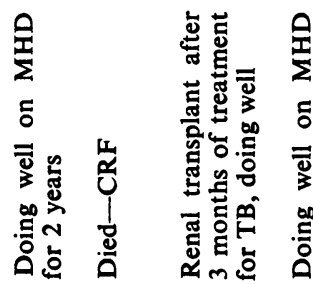

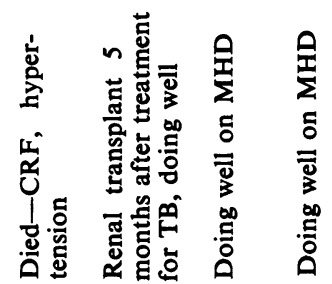

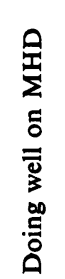

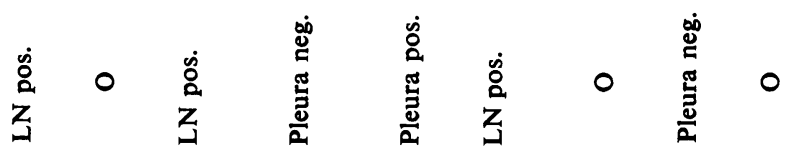

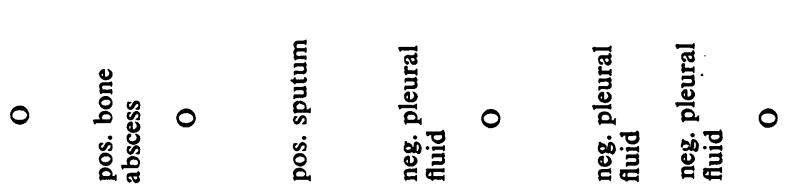

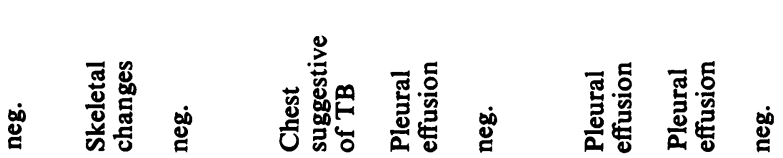

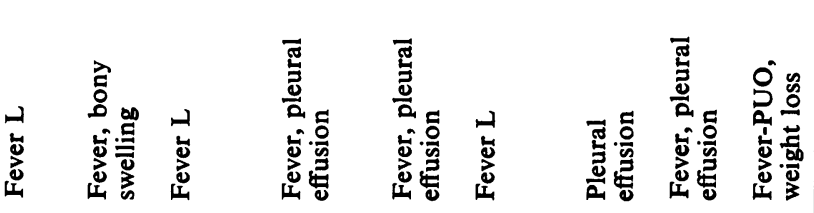

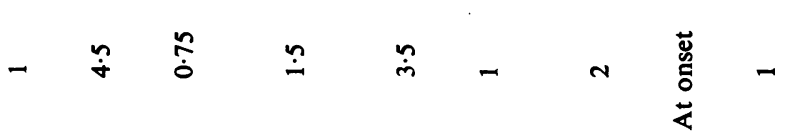

- $a$ m m $m$ \& 6

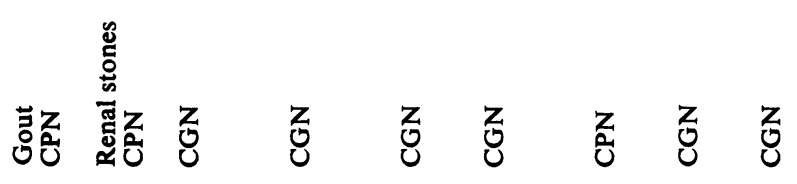

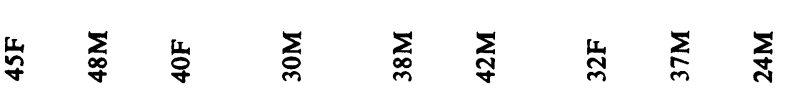

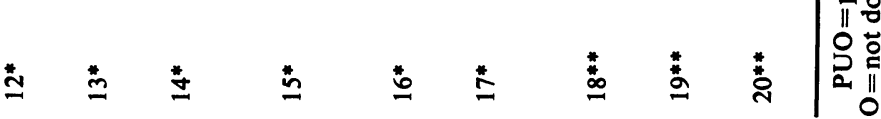


TABLE 2. Tuberculosis (TB) in maintenance haemodialysis patients-clinical features

\begin{tabular}{|c|c|c|c|}
\hline $\begin{array}{l}\text { Clinical features } \\
\text { (no. of cases) }\end{array}$ & $\begin{array}{l}\text { Details of } \\
\text { clinical features }\end{array}$ & $\begin{array}{l}\text { Other associated } \\
\text { clinical features }\end{array}$ & Special remarks \\
\hline Fever (15) & $\begin{array}{l}\text { Fever irregular } \\
\text { Occasionally very high }\end{array}$ & $\begin{array}{l}\text { Pleural effusion (6) } \\
\text { Lymphadenopathy (4) } \\
\text { Pulmonary abnormalities (2) }\end{array}$ & $\begin{array}{l}\text { PUO (3), therapeutic trial } \\
\text { pos. in all } 3\end{array}$ \\
\hline Pleural effusion (10) & $\begin{array}{l}\text { Exudative effusion } \\
\text { Unilateral (9) } \\
\text { Bilateral (1) }\end{array}$ & $\begin{array}{l}\text { Fever (6) } \\
\text { Lymphadenopathy* (1) } \\
\text { Military TB* (1) }\end{array}$ & $\begin{array}{l}\text { Pleural fluid cultures pos. (2) } \\
\text { Pleural biopsy pos. (2) } \\
\text { Sputum culture pos. (1) } \\
\text { Tubercles on mesentery (1) } \\
\text { Lymph-node biopsy pos. (1) } \\
\text { Therapeutic trial pos. (3) }\end{array}$ \\
\hline Lymphadenopathy (6) & $\begin{array}{l}\text { Cervical (5) } \\
\text { Axillary (1) }\end{array}$ & $\begin{array}{l}\text { Fever (4) } \\
\text { Pleural effusion (1) }\end{array}$ & Lymph node biopsy pos. (6) \\
\hline Pulmonary abnormalities (4) & $\begin{array}{l}\text { Typical (2) } \\
\text { Atypical (2) }\end{array}$ & $\begin{array}{l}\text { PUO ( } 2 \text { initially) } \\
\text { Pleural effusion (1) }\end{array}$ & $\begin{array}{l}\text { Sputum culture pos. (1) } \\
\text { Therapeutic trial pos. (2) } \\
\text { Reactivation of old TB (1) }\end{array}$ \\
\hline Ascites and hepatomegaly (2) & Exudative ascites & $\begin{array}{l}\text { Miliary tuberculosis (1) } \\
\text { Tubercles on mesentery on } \\
\text { pre-transplant laparotomy }\end{array}$ & Therapeutic trial pos. (1) \\
\hline Other features (4) & $\begin{array}{l}\text { Marked anorexia (2) } \\
\text { Abnormal weight loss (1) } \\
\text { Bony swelling (1) }\end{array}$ & $\begin{array}{l}\text { Both patients with anorexia } \\
\text { had hepatomegaly and } \\
\text { ascites }\end{array}$ & Bone abcess culture pos. (1) \\
\hline
\end{tabular}

*Biopsy/culture positive for tuberculosis; **Pleural biopsy could be done in 5 cases only; PUO=pyrexia of unknown origin $\overrightarrow{; \dot{1}}$ neg. = negative; pos. = positive.

TABLE 3. Tuberculosis in maintenance haemodialysis patients. Basis for diagnosis of tuberculosis

\begin{tabular}{ll}
\hline \multicolumn{1}{c}{ Method } & \multicolumn{1}{c}{ Diagnostic yield with number of cases } \\
\hline Histology & Lymph node* (6) Pleura (2) Mesentery (1) \\
$\begin{array}{l}\text { Bacteriology } \\
\text { (culture positive) }\end{array}$ & $\begin{array}{l}\text { Pleural fluid (2) Lymph node* (1) } \\
\text { Bone abscess (1) Sputum (1) }\end{array}$ \\
Therapeutic trial & Response to antitubercular drugs (7) \\
\hline $\begin{array}{l}\text { *In one case lymph node had evidence of tuberculosis both on culture } \\
\text { and histology. }\end{array}$
\end{tabular}

and Oettinger, 1977; Drueke et al., 1978; Lundin et al., 1979; Sasaki et al., 1979). The variable incidence of tuberculosis in different countries and communities can be ascribed to difference in environmental factors, availability of diagnostic and treatment facilities, acceptability of available facilities by the population and efficiency of treatment programmes and policies. Tuberculosis was evident in 11 among the 20 cases in this series either at the onset of dialysis treatment ( 3 cases) or within the first month of dialysis treatment (8 cases). Considering the high incidence of tuberculosis in the general population in India, it seems likely that environmental factors must have contributed to the high incidence of tuberculosis in the present MHD patients in addition to the role played by the uraemic state.
The existence of tuberculosis in chronic renaक failure may not always be easy to diagnose. The unusual presentations and atypical features cono tinue to baffle the clinicians. Proudfoot et al. (1969P have drawn attention to the cryptic form of miliar tuberculosis in adults. This type of tuberculosis. does not present the usual clinical and radiologicatr features of miliary tuberculosis and is missed in life quite frequently. Tuberculosis has undergone a con siderable change in its clinical presentation over the last 25 years from a comparatively acute and exten sive disease among the young to more chronic and less extensive disease among the elderly (Nagpal 1978).

The pattern of tuberculosis seen by the presen 5 authors was somewhat different from that reported in the literature. The most important clinicaf 
presentations of disease in the present series were fever, pleural effusion and lymphadenopathy. Hepatomegaly which was an important finding in the series of Lundin et al. (1979) and bone tuberculosis which was frequently found by Papadimitriou et al. (1979) seen infrequently by the present authors. Pulmonary abnormalities were found in only 4 cases in this study. The incidence of miliary tuberculosis was low in contrast to that of Sasaki et al. (1979). Two of the present patients had a past history of tuberculosis and it is likely that reactivation of the disease occurred in these after the onset of chronic renal failure.

Pyrexia in patients with chronic renal failure may sometimes pose a diagnostic challenge. The investigations for various recognized forms of infections may prove to be negative. Pyrexia of unknown origin (PUO), however, can be a major form of presentation in the cryptic form of tuberculosis (Proudfoot et al., 1969): 3 cases in that study presented as PUO; Amedia and Oettinger (1977) and Drueke et al. (1978) found PUO to be an important presentation of tuberculosis.

Pleural effusion in uraemics may arise as a result of circulatory congestion, uraemic pleuritis or nephrotic syndrome. Tubercular pleural effusion can, however, usually be distinguished from such pleural effusion by gross, microscopic, culture and chemical examination of pleural fluid. The clinical manifestations of tuberculosis in other organs and sites may be found in some cases. Pleural fluid AFB smear and culture was found to be positive in 30$70 \%$ and $10-70 \%$ of cases (Gryzbowski, 1968). Pleural biopsy may confirm the diagnosis in some of the patients as happened in 2 of the present cases.

Ascites was seen in 2 of the present cases but can occur in patients with renal failure without underlying infection (Cinque and Letteri, 1973). The protein content of ascitic fluid in such cases is usually less than $3 \mathrm{~g} / \mathrm{dl}$. The ascitic fluid is usually acellular or hypocellular and the cell count is rarely above $0.02 \times 10^{\circ} / 1$. Most patients with idiopathic ascites have a past history of peritoneal dialysis which may have produced chronic peritonitis.

There is no diagnostic problem in uraemic patients with superficial lymphadenopathy. Histopathological proof of tuberculosis is easily obtained in such patients as was the case in 6 patients in this study.

Tuberculosis was the cause of death in only $10 \%$ of cases in this study, although overall mortality was high for other reasons. Average mortality due to tuberculosis in MHD patients has been reported to be $11 \%$ (Papadimitriou et al., 1979).

The tuberculin skin test is negative in the majority of uraemics with tubercular infection; it was positive in only 5 of 15 patients in the present study. These observations are similar to those of Drueke et al. (1978) and Amedia and Oettinger (1977).

There have been differences of opinion regarding the use of antitubercular drugs, their dose and the duration of treatment in the management of tuberculosis in patients with renal failure. A combination of rifampicin and isoniazid is considered safe. Rifampicin is largely excreted in the bile and modification of its dose is not needed in renal failure. The risk of isoniazid therapy is well recognized, it is subject to acetylation in the liver and for slow acetylators a reduction of dose is necessary (Bowersox, 1973). It is good practice to restrict the dose of isoniazid to $200 \mathrm{mg} /$ day in patients with tuberculosis and end-stage renal disease. Ethambutol is excreted via the kidneys $80 \%$ unchanged. With renal failure its dose should be reduced to $5 \mathrm{mg} / \mathrm{kg} /$ day (Maher, 1978). Opinion is divided regarding optimum duration of antitubercular treatment; the chemotherapy, however, must be given for a period of at least 2 years.

Tuberculosis must be considered in clinical settings and geographic areas where there is a high incidence of tuberculosis. If the usual means of evaluation have been exhausted without revealing an aetiology for clinical features which could be tuberculosis in patients of chronic renal failure and the therapy with common antibiotics has failed, a trial of antitubercular chemotherapy is justified. Lundin et al. (1979) observed that the diagnosis and treatment of extrapulmonary tuberculosis may be delayed because of difficulty in obtaining positive cultures. This led to death of 2 of their patients in a group of 8 on maintenance haemodialysis having tuberculosis.

\section{References}

Amedia, C. \& Oetringer, C.W. (1977) Unusual presentation of tuberculosis in chronic hemodialysis patients. Clinical Nephrology, 8, 363.

Boulton-Jones, J.M., Vick, R., CAmeron, J.S. \& Black, P.J. (1973) Immune responses in uremia. Clinical Nephrology 1, 341.

Bowersox, D.W., Winterbauer, R.H., Steward, C.L., ORMER, B. \& BARRON, E. (1973) Isoniazid dosage in patients with renal failure. New England Journal of Medicine, 289, 84.

CinQue, T.J. \& LeTteri, J.M. (1973) Idiopathic ascites in chronic renal failure. New York State Journal of Medicine, 73, 781.

Dammin, C.J., Cough, N.P. \& Murray, J.E. (1957) Prolonged survival of skin homografts in uremic patients. Annals of the New York Academy of Sciences, 64, 967.

Drueke, T., Zingroff, J., François, Y., DiGiulio, S., MaN, N.K., Jungers, P. \& Crosnier, J. (1978) Tuberculose chez les urémiques traités par hémodialyse périodique (H.P.). Abstract. VIIth International Congress of Nephrology, Montreal, pp. 5-25.

Goyal, S.S., Mathur, G.P. \& PamRa, S.P. (1978) Tuberculosis trends in an urban community. Indian Journal of Tuberculosis, 25, 77.

GRYZBowSKI, S. (1968) Statement by the Committee on 
Therapy, American Thoracic Society: therapy of pleural effusion. American Review of Respiratory Disease, 97, 479.

Lundin, A.P., Alder, A.J., Berlyne, G.M. \& Friedman, E.A. (1979) Tuberculosis in patients undergoing maintenance hemodialysis. American Journal of Medicine, 67, 597.

Mackaness, G.B. (1971) Delayed hypersensitivity and the mechanism of cellular resistance to infection. In: Progress in Immunology (Ed by Amos, B.), p. 413. Academic Press, Inc., New York.

MAHER, J.F. (1978) Pharmacological aspects of regular dialysis treatment. In: Replacement of renal function (Ed by Drukker, W., Parsons, F.M. \& Maher, J.F.), p. 384. Nijhoff, The Hague.

Malaviya, A.N., Kumar, R., Kathpalia, S.C. \& Malhotra, K.K. (1973) Non-specific cellular and specific cell-mediated immunological responsiveness in chronic renal failure. Indian Journal of Medical Research, 61, 1194.

MuNT, R.W. (1971) Miliary tuberculosis in the chemotherapy era: with a clinical review in 69 American adults. Medicine. Baltimore, 51, 139.

NAGPAL, D.R. (1978) Tuberculosis in India-a perspective. Journal of the Indian Medical Association, 71, 44.

NAKHLA, L.S. \& Goggin, M.L. (1973) Lymphocyte transformation in chronic renal failure. Immunology, 24, 229.
National Tuberculosis Institute (1974) Tuberculosis in rural population of South India: A five-year epidemi logical study. Bulletin of the World Health Organization, $51,473$.

Ogg, C.S., Toseland, P.A. \& Cameron, J.S. (1968) Pư monary tuberculosis in a patient on intermittent haem dialysis. British Medical Journal, 2, 283.

Papadimitriou, M., Memmos, D. \& Metaxas, P. (197 Tuberculosis in patients on maintenance haemodialys Nephron, 24, 53.

Pradhan, R.P., Katz, L.A., Nidus, B.D., Matalon, R. EISINGER, R.P. (1974) Tuberculosis in dialyzed patients. Journal of the American Medical Association, 229, 798.

Proudfoot, A.T., Akhtar, A.J., Douglas, S.C. \& HorNE N.W. (1969) Miliary tuberculosis in adults. British Medicg: Journal, 2, 273.

Sasaki, S., Akiba, T., Matsuhiko, S., Tomura, S., Yos yama, N., Nakagawa, S., Shoji, T., Takuo, S. \& Tak UCHI, J. (1979) Ten years survey of dialysis-associated tuberculosis. Nephron, 24, 141.

Wilson, W.E.C., Kirkpatrick, C.H. \& Talmage, D. (1965) Suppression of immunologic responsiveness uremia. Annals of Internal Medicine, 62, 1. 